[Article]

\title{
$\boldsymbol{\alpha}$-氨基丁酸对映异构体在 DMF+水混合溶剂中的焓对作用
}

\author{
郭 政 胡新根 ${ }^{*}$ 梁红玉 贾召鹏 程维娜 刘嘉敏
}

(温州大学化学与材料工程学院, 浙江 温州 325035)

\begin{abstract}
摘要: 利用等温滴定微量热法(ITC)分别测定了 $298.15 \mathrm{~K}$ 时 L- $\alpha$-氨基丁酸和 D- $\alpha$-氨基丁酸两种对映异构体在 不同组成的二甲基甲酰胺(DMF)+水混合溶剂中的稀释焓. 根据统计热力学的 McMillan-Mayer 理论计算各溶 剂组成下的同系焓对作用系数 $\left(h_{x x}\right)$. 从溶质 - 溶质和溶质 - 溶剂相互作用的观点出发探讨了三元水溶液中疏 水一疏水、疏水-亲水和亲水-亲水作用的竞争平衡. 实验发现, 在所研究的混合溶剂组成范围内 $\left(w_{\mathrm{DMF}}=0-0.3\right)$, $\alpha$-氨基丁酸两种对映体的 $h_{\mathrm{x}}$ 都是较大的正值, 且都随 $W_{\mathrm{DM}}$ 的增大而逐渐减小, 而有趣的是 $\mathrm{L}$ 型对映体的 $h_{\mathrm{xx}}$ 值 普遍比 $\mathrm{D}$ 型的大 $\left(h_{\mathrm{L}}>h_{\mathrm{DD}}\right)$, 说明 ITC 可以区分对映异构体的同手性焓对作用. 结果表明: 在 $\alpha$-氨基丁酸+水 + $\mathrm{DMF}$ 三元溶液体系中, 疏水-疏水和疏水-亲水作用在分子对作用过程中占优势; 在 $\alpha$-氨基丁酸的同种对映体 发生分子对作用时, L-L 分子对比 D-D 分子对在构型上更有利于 $\alpha$-碳上疏水侧链 $\left(\mathrm{CH}_{3} \mathrm{CH}_{2}-\right)$ 的靠拢, 疏水基 团的水合壳层发生交盖, 局部破坏而释放出部分结构化的水, 过程自发、吸热且伴随显著摘增 $(\Delta G<0, \Delta H>0$, $\Delta S=(\Delta H-\Delta G) / T>0)$, 因而在稀释时释放出更多热量, $h_{x x}$ 具有较大的正值.
\end{abstract}

关键词： $\alpha$-氨基丁酸; 对映异构体； DMF+水混合溶剂；等温滴定微量热法；稀释焓；焓对作用系数； 手性区分效应

中图分类号: 0645

\section{Enthalpic Pairwise Interactions of $\alpha$-Aminobutyric Acid Enantiomers in DMF+Water Mixtures}

\author{
GUO Zheng \\ HU Xin-Gen* LIANG Hong-Yu \\ JIA Zhao-Peng \\ CHENG Wei-Na LIU Jia-Min
}

(College of Chemistry and Materials Engineering, Wenzhou University, Wenzhou 325035, Zhejiang Province, P. R. China)

\begin{abstract}
Dilution enthalpies of two $\alpha$-aminobutyric acid enantiomers, L- $\alpha$-aminobutyric acid and D- $\alpha$-aminobutyric acid, in dimethylformide (DMF)+water mixtures of various compositions are determined by isothermal titration microcalorimetry (ITC) at $298.15 \mathrm{~K}$. Homotactic enthalpic pairwise interaction coefficients for each solvent composition are calculated according to the McMillan-Mayer theory of statistical thermodynamics. From the point of view of solute-solute and solute-solvent interactions, competition equilibria among hydrophobic-hydrophobic, hydrophobic-hydrophilic, and hydrophilichydrophilic interactions in ternary solutions are explored. It is found that all values of $h_{x x}$ are positive across the entire studied composition range of mixed solvents (mass fraction of $D M F, w_{D M F}=0-0.3$ ), gradually reducing with the increasing $W_{\text {DMF. }}$ It is of interest that the $h_{x \mathrm{x}}$ values for the L-enantiomer are universally larger than those of the D-enantiomer $\left(h_{\llcorner}>h_{\mathrm{D} D}\right)$, which indicates that ITC is useful to discriminate homochiral enthalpic pairwise interaction of enantiomers. Our results show that hydrophobic-hydrophobic and hydrophobic-hydrophilic interactions are predominant in pairwise molecular interaction processes in ternary solutions containing $\alpha$-aminobutyric acid, water, and DMF, and that the configuration of $\mathrm{L}-\mathrm{L}$ molecular pair is more advantageous for the approach of hydrophobic side-chains $\left(\mathrm{CH}_{3} \mathrm{CH}_{2}-\right)$ on $\alpha$-carbon
\end{abstract}

Received: March 17, 2012; Revised: June 25, 2012; Published on Web: June 26, 2012.

"Corresponding author. Email: hxgwzu@126.com; Tel: +86-577-86596022.

The project was supported by the National Natural Science Foundation of China (21073132).

国家自然科学基金(21073132)资助项目

(C) Editorial office of Acta Physico-Chimica Sinica 
than a D-D pair, where part of the structured water molecules relax to less structured bulky water due to the overlap and partial breaking of hydrophobic hydration cospheres around nonpolar groups. This confirms that the process is spontaneous and is accompanied with positive enthalpy change and obvious increase in entropy $(\Delta G<0, \Delta H>0$, and $\Delta S=(\Delta H-\Delta G) / T>0)$, consequently releasing more heat upon dilution of the solutions and leading to larger values of $h_{x x}$.

Key Words: $\quad \alpha$-Aminobutyric acid; Enantiomer; DMF+water mixture; Isothermal ditration microcalorimetry; Dilution enthalpy; Enthalpic pairwise interaction; Chirality discrimination effect

\section{1 引言}

溶液中的溶质-溶质相互作用常根据 McMillanMayer 统计热力学理论进行研究 ${ }^{1.2}$ 利用各种微量热 法测定溶解焓、稀释焓或混合焓, 可以计算得到溶 质的同系或异系焓对作用系数 $\left(h_{\mathrm{xx}}\right.$ 或 $\left.h_{\mathrm{x} y}\right){ }^{3-5}$ 焓对作 用系数被认为是溶液中溶剂介入的溶质 - 溶质相互 作用焓效应的一种有效度量. ${ }^{67}$ Castronuovo 等 ${ }^{8}$ 通过 对一系列二元烷醇在乙醇+水混合溶剂中同系焓对 作用系数的测定, 考察了共溶剂的存在对疏水作用 的影响. Palecz ${ }^{9,10}$ 通过对一系列 $\mathrm{L}-\alpha$-氨基酸同系和 交叉焓对作用的研究, 建立了一种描述氨基酸疏水 性的热力学尺度. 我们 ${ }^{11-13}$ 利用微量热法测定了一 些典型 $\alpha$-氨基酸和手性二元醇在二甲亚砜(DMSO) 水溶液中的稀释焓, 探讨了焓对作用的溶剂效应, 发现了不同对映异构体同手性对作用的焓区分效 应, 即 S-型或 L-型异构体的 $h_{x x}$ 普遍要比 R-型或 D型异构体的大. 为了深入研究溶液中对映异构体分 子焓对作用的手性区分效应, 我们选择了在 $\alpha$-碳上 带有较大疏水侧链的 $\alpha$-氨基酸对映异构体, 即 $\mathrm{L}-\alpha-$ 氨基丁酸和 $\mathrm{D}-\alpha$-氨基丁酸作为研究对象, 以 $N, N-$ 二 甲基甲酰胺(DMF)作为共溶剂, 探讨水溶液环境下 疏水性取代基和强极性共溶剂对 $\alpha$-氨基酸同手性焓 对作用的影响.

\section{2 实 验}

\section{1 试剂和仪器}

$\mathrm{L}-\alpha$-氨基丁酸和 $\mathrm{D}-\alpha$-氨基丁酸购自日本 $\mathrm{TCI}$ 公 司, 纯度均在 $99 \%$ 以上. 样品在使用前除了干燥外, 未作进一步提纯处理. DMF 用 $4 \mathrm{~A}$ 分子篮干燥过夜 后, 减压蒸馏收集 $314.25 \mathrm{~K}$ 下的馏分干燥 $24 \mathrm{~h}$ 备 用. 混合溶剂和溶液的配制都采用重量法, 使用十 万分之一天平, 误差 $\pm 0.1 \mathrm{mg}$. 实验用水为 Milli- $Q$ 高 纯水, 使用 Millipore Elix5/Milli-Q系统制备.

溶液稀释焓的测定是利用美国 MicroCal 公司
新一代的等温滴定微量热仪 ITC200. 测量池和参比 池体积为 $200 \mu \mathrm{L}$, 注射器体积为 $40 \mu \mathrm{L}$, 每次注射时 每滴样品体积最小可达 $0.1 \mu \mathrm{L}$.

\section{2 实验和数据处理方法}

采用连续注射的方法测定样品溶液的稀释焓. 被稀释的溶液(纯水 $+\alpha$-氨基丁酸二元溶液或纯水 + $\mathrm{DMF}+\alpha$-氨基丁酸三元溶液)装在注射器中, 在测量 池和参比池中装组成相同的溶剂(纯水或 $\mathrm{DMF}+$ 水 混合溶剂). 在注射开始前, 溶液和溶剂都要进行赶 气泡处理.

测量池温度控制在 $298.15 \mathrm{~K}$. 总的注射次数设 定为 20 次, 每次 $2 \mu \mathrm{L}$; 每次注射持续时间为 $4 \mathrm{~s}$, 连 续注射的时间间隔为 $120 \mathrm{~s}$; 摚拌速度控制为 1000 $r \cdot \mathrm{min}^{-1}$. ITC 热转变曲线由仪器自带的 Origin 计算 软件处理生成.

实验数据处理的基本原理及方法参见文献. ${ }^{11-13}$ 在各溶剂组成下的同系焓对作用系数 $h_{\mathrm{xx}}$ 的计算可 根据式(1)由第 $N$ 次注射的摩尔稀释焓 $\Delta H\left(m_{N-1} \rightarrow m_{N}\right)$ 对 $N$ 进行线性回归分析得到. ${ }^{14}$

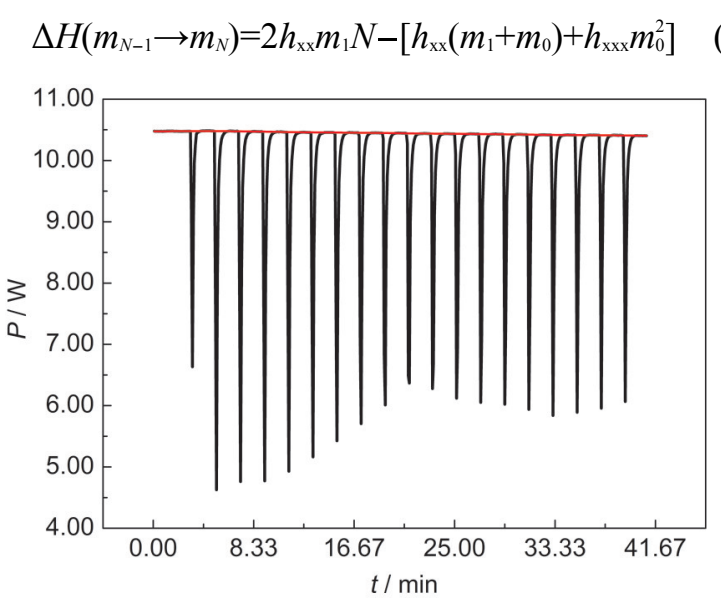

图 $1298.15 \mathrm{~K}$ 时 $\mathrm{L}-\alpha$-氨基丁酸在纯水中稀释的 ITC 热转 变(热功率 $(P)-$ 时间 $(t))$ 曲线

Fig.1 ITC thermal transition (thermal power $(P)$-time $(t)$ ) curve of $L$ - $\alpha$-aminobutyric acid diluted in pure water at $298.15 \mathrm{~K}$ 
表 $1298.15 \mathrm{~K}$ 时 $\mathrm{L}-\boldsymbol{\alpha}$-氨基丁酸和 D- $\alpha$-氨基丁酸在纯水中的稀释焓

Table 1 Experimental dilution enthalpies of $\mathrm{L}$ - $\alpha$-aminobutyric acid and D- $\alpha$-aminobutyric acid in pure water at $298.15 \mathrm{~K}$

\begin{tabular}{|c|c|c|c|c|c|c|c|}
\hline \multicolumn{4}{|c|}{ L- $\alpha$-aminobutyric acid } & \multicolumn{4}{|c|}{ D- $\alpha$-aminobutyric acid } \\
\hline$N$ & $m_{N-1} /\left(\mathrm{mol} \cdot \mathrm{kg}^{-1}\right)$ & $m_{N} /\left(\mathrm{mol} \cdot \mathrm{kg}^{-1}\right)$ & $\Delta H\left(m_{N-1} \rightarrow m_{N}\right) /\left(\mathrm{J} \cdot \mathrm{mol}^{-1}\right)$ & $N$ & $m_{N-1} /\left(\mathrm{mol} \cdot \mathrm{kg}^{-1}\right)$ & $m_{N} /\left(\mathrm{mol} \cdot \mathrm{kg}^{-1}\right)$ & $\Delta H\left(m_{N-1} \rightarrow m_{N}\right) /\left(\mathrm{J} \cdot \mathrm{mol}^{-1}\right)$ \\
\hline 2 & 0.00256 & 0.00764 & $-161.56(1.86)$ & 2 & 0.00250 & 0.00745 & $-171.36(1.35)$ \\
\hline 3 & 0.00764 & 0.01266 & $-262.48(0.00)$ & 3 & 0.00745 & 0.01235 & $-252.67(-0.03)$ \\
\hline 4 & 0.01266 & 0.01764 & $-257.81(-0.26)$ & 4 & 0.01235 & 0.01720 & $-247.14(0.34)$ \\
\hline 5 & 0.01764 & 0.02256 & $-251.88(-0.34)$ & 5 & 0.01720 & 0.02201 & $-241.15(0.70)$ \\
\hline 6 & 0.02256 & 0.02744 & $-245.12(0.23)$ & 6 & 0.02201 & 0.02676 & $-234.73(0.41)$ \\
\hline 7 & 0.02744 & 0.03226 & $-240.19(-0.13)$ & 7 & 0.02676 & 0.03146 & $-230.35(0.21)$ \\
\hline 8 & 0.03226 & 0.03703 & $-235.65(0.02)$ & 8 & 0.03146 & 0.03612 & $-225.84(0.34)$ \\
\hline 9 & 0.03703 & 0.04175 & $-231.28(-0.21)$ & 9 & 0.03612 & 0.04072 & $-221.76(0.14)$ \\
\hline 10 & 0.04175 & 0.04642 & $-226.48(-0.17)$ & 10 & 0.04072 & 0.04527 & $-217.11(0.36)$ \\
\hline 11 & 0.04642 & 0.05104 & $-222.06(-0.19)$ & 11 & 0.04527 & 0.04978 & $-213.51(0.32)$ \\
\hline 12 & 0.05104 & 0.05561 & $-217.27(-0.05)$ & 12 & 0.04978 & 0.05423 & $-209.65(0.04)$ \\
\hline 13 & 0.05561 & 0.06012 & $-213.43(0.14)$ & 13 & 0.05423 & 0.05864 & $-206.07(0.18)$ \\
\hline 14 & 0.06012 & 0.06459 & $-209.19(0.21)$ & 14 & 0.05864 & 0.06299 & $-202.31(0.31)$ \\
\hline 15 & 0.06459 & 0.06900 & $-204.61(0.23)$ & 15 & 0.06299 & 0.06730 & $-198.00(0.43)$ \\
\hline 16 & 0.06900 & 0.07336 & $-201.03(0.13)$ & 16 & 0.06730 & 0.07155 & $-194.23(0.28)$ \\
\hline 17 & 0.07336 & 0.07767 & $-197.19(0.14)$ & 17 & 0.07155 & 0.07576 & $-190.84(0.13)$ \\
\hline 18 & 0.07767 & 0.08194 & $-194.06(0.18)$ & 18 & 0.07576 & 0.07991 & $-187.96(0.34)$ \\
\hline 19 & 0.08194 & 0.08614 & $-190.15(-0.08)$ & 19 & 0.07991 & 0.08402 & $-184.61(0.41)$ \\
\hline 20 & 0.08614 & 0.09030 & $-186.73(0.01)$ & 20 & 0.08402 & 0.08807 & $-180.54(0.16)$ \\
\hline
\end{tabular}

The values in parentheses are the evaluated uncertainties $\left(\sigma_{\mathrm{H}}\right)$ of $\Delta H\left(m_{N-1} \rightarrow m_{N}\right), \sigma_{\mathrm{H}}=100[\Delta H($ expt. $)-\Delta H($ calc. $)] / \Delta H($ expt. $)$, in which $\Delta H$ (expt.) represents the experimental value of $\Delta H\left(m_{N-1} \rightarrow m_{N}\right)$, and $\Delta H$ (calc.) represents the calculated value of $\Delta H\left(m_{N-1} \rightarrow m_{N}\right)$.

式中, $m_{N-1}$ 和 $m_{N}$ 分别表示第 $N-1$ 次和第 $N$ 次注射稀 释后测量池中溶液的质量摩尔浓度, $m_{0}$ 为稀释前的 溶液原始浓度, $h_{\mathrm{xxx}}$ 为焓三作用系数.

ITC 用于稀释焓测定的精确度经实验验证符合 要求. ${ }^{13}$ 所有样品都在同一条件下平行测定三次, 最 后计算 $h_{\mathrm{xx}}$ 的平均值.

\section{3 结果与讨论}

我们以 $\mathrm{L}-\alpha$-氨基丁酸 $298.15 \mathrm{~K}$ 时在纯水中的稀

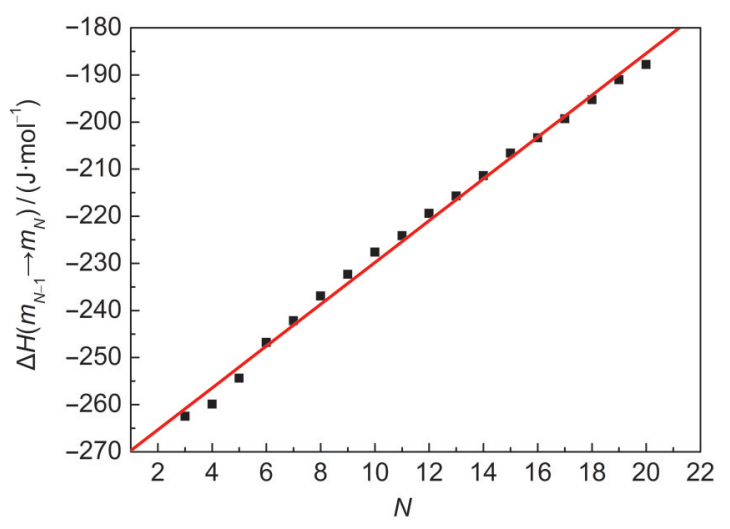

图 $2 \mathrm{~L}-\alpha$-氨基丁酸 $298.15 \mathrm{~K}$ 时在纯水中的稀释焓以滴定次 数 $N$ 为函数的拟合值 $\left(\Delta H\left(m_{N-1} \rightarrow m_{N}\right)\right)$

Fig.2 Fitting experimental values of $\Delta H\left(m_{N-1} \rightarrow m_{N}\right)$ of $\mathrm{L}$ - $\alpha$-aminobutyric acid in pure water as a function of injection number $N$ at $298.15 \mathrm{~K}$
释焓为例, 把它的原始实验滴定曲线绘于图 1 , 把稀 释焓 $\Delta H\left(m_{N-1} \rightarrow m_{N}\right)$ 以滴定次数 $N$ 为函数的拟合曲线 绘于图 2.

$\mathrm{L}-\alpha$-氨基丁酸和 $\mathrm{D}-\alpha$-氨基丁酸在纯水中的稀释 焓数据列于表 1 , 在不同质量分数的 $\mathrm{DMF}+$ 水混合 溶剂中的稀释焓数据列于表 2. 根据式(1)回归分析 得到的同系焓对作用系数值 $h_{\mathrm{xx}}$ 列于表 3 , 相关系数 的平方 $R^{2}$ 均大于 0.95 . 两种对映异构体的 $h_{\mathrm{xx}}$ 随混合

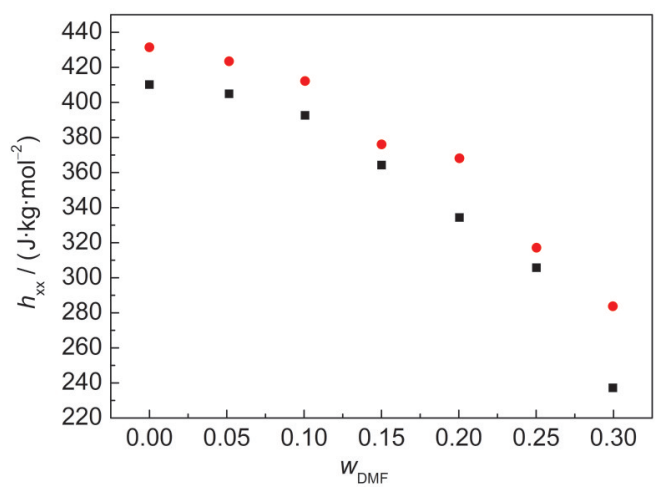

图 $3298.15 \mathrm{~K}$ 时 $\mathrm{L}-\alpha$-氨基丁酸和 D- $\alpha$-氨基丁酸在不同 DMF 质量分数的 DMF $+\mathrm{H}_{2} \mathrm{O}$ 混合溶剂中的焓对作用系数 $\left(h_{\mathrm{xx}}\right)$ 比较

Fig.3 Comparison of enthalpic pairwise interaction coefficients $\left(h_{\mathrm{xx}}\right)$ of D- $\alpha$-aminobutyric acid and

L- $\alpha$-aminobutyric acid in DMF+water mixtures with different mass fractions of DMF at 298.15 K ๑: L- $\alpha$-aminobutyric acid; $\mathbf{- 1}$ D- $\alpha$-aminobutyric acid 
表 $2298.15 \mathrm{~K}$ 时 $\mathrm{L}-\alpha$-氨基丁酸和 D- $\alpha$-氨基丁酸在不同 DMF 质量分数 $\left(w_{\mathrm{DMF}}\right.$ ) 的 DMF $+\mathrm{H}_{2} \mathrm{O}$ 混合溶剂中稀释焓的实验值 Table 2 Experimental dilution enthalpies of $L$ - $\alpha$-aminobutyric acid and D- $\alpha$-aminobutyric acid in DMF+water mixed solvents with different mass fractions of DMF ( $\left.w_{\text {DMF }}\right)$ at $298.15 \mathrm{~K}$

\begin{tabular}{|c|c|c|c|c|c|c|c|}
\hline$N$ & $m_{N-1} /\left(\mathrm{mol} \cdot \mathrm{kg}^{-1}\right)$ & $m_{N} /\left(\mathrm{mol} \cdot \mathrm{kg}^{-1}\right)$ & $\Delta H\left(m_{N-1} \rightarrow m_{N}\right) /\left(\mathrm{J} \cdot \mathrm{mol}^{-1}\right)$ & $N$ & $m_{N-1} /\left(\mathrm{mol} \cdot \mathrm{kg}^{-1}\right)$ & $m_{N} /\left(\mathrm{mol} \cdot \mathrm{kg}^{-1}\right)$ & $\Delta H\left(m_{N-1} \rightarrow m_{N}\right) /\left(\mathrm{J} \cdot \mathrm{mol}^{-1}\right)$ \\
\hline \multicolumn{8}{|c|}{ L- $\alpha$-aminobutyric acid } \\
\hline & & $w_{\mathrm{DMF}}=0.0515$ & & & & $w_{\mathrm{DMF}}=0.1001$ & \\
\hline 2 & 0.00243 & 0.00724 & $-135.80(3.43)$ & 2 & 0.00249 & 0.00744 & $-180.75(0.94)$ \\
\hline 3 & 0.00724 & 0.01201 & $-251.50(-0.32)$ & 3 & 0.00744 & 0.01233 & $-255.67(1.19)$ \\
\hline 4 & 0.01201 & 0.01673 & $-246.17(-0.22)$ & 4 & 0.01233 & 0.01718 & $-253.42(1.69)$ \\
\hline 5 & 0.01673 & 0.02141 & $-240.56(-0.18)$ & 5 & 0.01718 & 0.02197 & $-248.10(2.28)$ \\
\hline 6 & 0.02141 & 0.02603 & $-236.22(0.31)$ & 6 & 0.02197 & 0.02672 & $-240.49(1.76)$ \\
\hline 7 & 0.02603 & 0.03061 & $-231.90(0.33)$ & 7 & 0.02672 & 0.03141 & $-236.89(2.54)$ \\
\hline 8 & 0.03061 & 0.03513 & $-225.82(-0.03)$ & 8 & 0.03141 & 0.03606 & $-230.83(1.40)$ \\
\hline 9 & 0.03513 & 0.03961 & $-221.94(0.18)$ & 9 & 0.03606 & 0.04066 & $-225.14(1.76)$ \\
\hline 10 & 0.03961 & 0.04404 & $-217.60(-0.05)$ & 10 & 0.04066 & 0.04520 & $-222.05(1.34)$ \\
\hline 11 & 0.04404 & 0.04842 & $-213.12(0.03)$ & 11 & 0.04520 & 0.04970 & $-218.57(2.35)$ \\
\hline 12 & 0.04842 & 0.05275 & $-208.29(0.30)$ & 12 & 0.04970 & 0.05415 & $-214.20(2.18)$ \\
\hline 13 & 0.05275 & 0.05704 & $-204.90(0.21)$ & 13 & 0.05415 & 0.05854 & $-210.07(1.76)$ \\
\hline 14 & 0.05704 & 0.06127 & $-201.59(-0.01)$ & 14 & 0.05854 & 0.06289 & $-206.56(1.79)$ \\
\hline 15 & 0.06127 & 0.06546 & $-197.85(-0.10)$ & 15 & 0.06289 & 0.06719 & $-202.91(2.12)$ \\
\hline 16 & 0.06546 & 0.06960 & $-194.19(-0.05)$ & 16 & 0.06719 & 0.07144 & $-200.92(1.77)$ \\
\hline 17 & 0.06960 & 0.07369 & $-190.71(-0.01)$ & 17 & 0.07144 & 0.07564 & $-197.16(1.68)$ \\
\hline 18 & 0.07369 & 0.07773 & $-187.66(-0.11)$ & 18 & 0.07564 & 0.07979 & $-193.37(1.89)$ \\
\hline 19 & 0.07773 & 0.08173 & $-184.13(-0.11)$ & 19 & 0.07979 & 0.08388 & $-189.25(2.12)$ \\
\hline \multirow[t]{2}{*}{20} & 0.08173 & 0.08567 & $-180.31(0.11)$ & 20 & 0.08388 & 0.08793 & $-184.92(2.16)$ \\
\hline & & $w_{\text {DMF }}=0.1509$ & & & & $w_{\text {DMF }}=0.1995$ & \\
\hline 2 & 0.00250 & 0.00748 & $-168.37(0.39)$ & 2 & 0.00248 & 0.00741 & $-158.81(2.01)$ \\
\hline 3 & 0.00748 & 0.01240 & $-249.69(-0.50)$ & 3 & 0.00741 & 0.01228 & $-232.21(-2.12)$ \\
\hline 4 & 0.01240 & 0.01727 & $-246.77(-0.20)$ & 4 & 0.01228 & 0.01711 & $-227.92(-3.10)$ \\
\hline 5 & 0.01727 & 0.02209 & $-242.15(0.09)$ & 5 & 0.01711 & 0.02189 & $-224.31(-2.51)$ \\
\hline 6 & 0.02209 & 0.02686 & $-237.40(-0.16)$ & 6 & 0.02189 & 0.02662 & $-220.62(-2.18)$ \\
\hline 7 & 0.02686 & 0.03158 & $-233.21(0.33)$ & 7 & 0.02662 & 0.03129 & $-216.76(-2.67)$ \\
\hline 8 & 0.03158 & 0.03626 & $-229.00(0.23)$ & 8 & 0.03129 & 0.03592 & $-211.37(-1.92)$ \\
\hline 9 & 0.03626 & 0.04088 & $-225.42(0.05)$ & 9 & 0.03592 & 0.04050 & $-208.07(-1.77)$ \\
\hline 10 & 0.04088 & 0.04545 & $-220.87(-0.45)$ & 10 & 0.04050 & 0.04503 & $-204.15(-1.39)$ \\
\hline 11 & 0.04545 & 0.04997 & $-218.02(0.49)$ & 11 & 0.04503 & 0.04951 & $-200.09(-1.36)$ \\
\hline 12 & 0.04997 & 0.05444 & $-213.30(0.09)$ & 12 & 0.04951 & 0.05394 & $-195.59(-1.75)$ \\
\hline 13 & 0.05444 & 0.05886 & $-210.56(0.28)$ & 13 & 0.05394 & 0.05832 & $-192.25(-1.80)$ \\
\hline 14 & 0.05886 & 0.06323 & $-206.74(0.49)$ & 14 & 0.05832 & 0.06265 & $-188.60(-1.84)$ \\
\hline 15 & 0.06323 & 0.06756 & $-202.85(0.12)$ & 15 & 0.06265 & 0.06694 & $-185.32(-1.25)$ \\
\hline 16 & 0.06756 & 0.07183 & $-199.53(-0.08)$ & 16 & 0.06694 & 0.07117 & $-182.02(-1.45)$ \\
\hline 17 & 0.07183 & 0.07605 & $-195.66(-0.14)$ & 17 & 0.07117 & 0.07535 & $-178.38(-1.48)$ \\
\hline 18 & 0.07605 & 0.08022 & $-192.61(-0.42)$ & 18 & 0.07535 & 0.07948 & $-175.23(-1.66)$ \\
\hline 19 & 0.08022 & 0.08434 & $-189.23(-0.54)$ & 19 & 0.07948 & 0.08357 & $-172.26(-1.94)$ \\
\hline \multirow[t]{2}{*}{20} & 0.08434 & 0.08841 & $-185.33(-0.53)$ & 20 & 0.08357 & 0.08760 & $-168.77(-1.60)$ \\
\hline & & $w_{\text {DMF }}=0.2502$ & & & & $w_{\text {DMF }}=0.2998$ & \\
\hline 2 & 0.00251 & 0.00748 & $-143.56(7.86)$ & 2 & 0.00221 & 0.0066 & $-102.47(-2.06)$ \\
\hline 3 & 0.00748 & 0.01240 & $-208.23(2.35)$ & 3 & 0.00660 & 0.01095 & $-164.95(-0.02)$ \\
\hline 4 & 0.01240 & 0.01728 & $-209.76(1.20)$ & 4 & 0.01095 & 0.01525 & $-166.43(-0.12)$ \\
\hline 5 & 0.01728 & 0.02210 & $-202.12(2.90)$ & 5 & 0.01525 & 0.01951 & $-162.87(-1.82)$ \\
\hline 6 & 0.02210 & 0.02687 & $-196.40(2.78)$ & 6 & 0.01951 & 0.02373 & $-162.97(-2.68)$ \\
\hline 7 & 0.02687 & 0.03160 & $-199.11(-0.11)$ & 7 & 0.02373 & 0.02790 & $-158.51(-1.40)$ \\
\hline 8 & 0.03160 & 0.03627 & $-195.29(-0.23)$ & 8 & 0.02790 & 0.03203 & $-154.62(-1.37)$ \\
\hline 9 & 0.03627 & 0.04089 & $-192.09(-0.13)$ & 9 & 0.03203 & 0.03611 & $-152.61(-1.71)$ \\
\hline 10 & 0.04089 & 0.04547 & $-189.24(-0.41)$ & 10 & 0.03611 & 0.04015 & $-149.99(-1.62)$ \\
\hline 11 & 0.04547 & 0.04999 & $-187.81(-1.48)$ & 11 & 0.04015 & 0.04414 & $-147.57(-1.60)$ \\
\hline
\end{tabular}


continued Table 2

\begin{tabular}{|c|c|c|c|c|c|c|c|}
\hline$N$ & $m_{N-1} /\left(\mathrm{mol} \cdot \mathrm{kg}^{-1}\right)$ & $m_{N} /\left(\mathrm{mol} \cdot \mathrm{kg}^{-1}\right)$ & $\Delta H\left(m_{N-1} \rightarrow m_{N}\right) /\left(\mathrm{J} \cdot \mathrm{mol}^{-1}\right)$ & $N$ & $m_{N-1} /\left(\mathrm{mol} \cdot \mathrm{kg}^{-1}\right)$ & $m_{N} /\left(\mathrm{mol} \cdot \mathrm{kg}^{-1}\right)$ & $\Delta H\left(m_{N-1} \rightarrow m_{N}\right) /\left(\mathrm{J} \cdot \mathrm{mol}^{-1}\right)$ \\
\hline 12 & 0.04999 & 0.05446 & $-180.23(0.47)$ & 12 & 0.04414 & 0.04809 & $-144.91(-1.68)$ \\
\hline 13 & 0.05446 & 0.05889 & $-178.88(-0.22)$ & 13 & 0.04809 & 0.05200 & $-142.51(-2.27)$ \\
\hline 14 & 0.05889 & 0.06326 & $-174.59(0.40)$ & 14 & 0.05200 & 0.05586 & $-140.10(-2.37)$ \\
\hline 15 & 0.06326 & 0.06758 & $-171.23(0.35)$ & 15 & 0.05586 & 0.05967 & $-137.43(-1.96)$ \\
\hline 16 & 0.06758 & 0.07185 & $-168.43(0.48)$ & 16 & 0.05967 & 0.06345 & $-133.65(-0.83)$ \\
\hline 17 & 0.07185 & 0.07608 & $-165.26(0.77)$ & 17 & 0.06345 & 0.06718 & $-132.41(-1.87)$ \\
\hline 18 & 0.07608 & 0.08025 & $-163.32(0.00)$ & 18 & 0.06718 & 0.07086 & $-129.87(-1.74)$ \\
\hline 19 & 0.08025 & 0.08437 & $-159.46(-0.41)$ & 19 & 0.07086 & 0.07450 & $-127.44(-1.82)$ \\
\hline 20 & 0.08437 & 0.08845 & $-156.71(0.16)$ & 20 & 0.07450 & 0.07810 & $-125.73(-1.75)$ \\
\hline \multicolumn{8}{|c|}{ D- $\alpha$-aminobutyric acid } \\
\hline \multicolumn{4}{|c|}{$w_{\text {DMF }}=0.0515$} & \multicolumn{4}{|c|}{$w_{\mathrm{DMF}}=0.1001$} \\
\hline 2 & 0.00248 & 0.00739 & $-172.52(-0.30)$ & 2 & 0.00251 & 0.00751 & $-165.67(-3.41)$ \\
\hline 3 & 0.00739 & 0.01226 & $-253.74(0.10)$ & 3 & 0.00751 & 0.01245 & $-256.32(0.02)$ \\
\hline 4 & 0.01226 & 0.01707 & $-249.79(0.57)$ & 4 & 0.01245 & 0.01734 & $-253.93(-0.01)$ \\
\hline 5 & 0.01707 & 0.02184 & $-244.08(0.28)$ & 5 & 0.01734 & 0.02218 & $-247.57(0.19)$ \\
\hline 6 & 0.02184 & 0.02656 & $-240.12(0.32)$ & 6 & 0.02218 & 0.02697 & $-242.13(-0.03)$ \\
\hline 7 & 0.02656 & 0.03122 & $-234.07(0.96)$ & 7 & 0.02697 & 0.03171 & $-238.32(-0.04)$ \\
\hline 8 & 0.03122 & 0.03584 & $-228.45(1.06)$ & 8 & 0.03171 & 0.03640 & $-233.16(0.36)$ \\
\hline 9 & 0.03584 & 0.04041 & $-226.92(0.35)$ & 9 & 0.03640 & 0.04104 & $-234.16(-1.94)$ \\
\hline 10 & 0.04041 & 0.04493 & $-222.48(-0.05)$ & 10 & 0.04104 & 0.04563 & $-225.69(-0.09)$ \\
\hline 11 & 0.04493 & 0.04940 & $-217.55(0.24)$ & 11 & 0.04563 & 0.05017 & $-221.50(0.00)$ \\
\hline 12 & 0.04940 & 0.05382 & $-211.95(0.27)$ & 12 & 0.05017 & 0.05465 & $-217.05(-0.21)$ \\
\hline 13 & 0.05382 & 0.05819 & $-208.62(0.38)$ & 13 & 0.05465 & 0.05909 & $-214.18(-0.49)$ \\
\hline 14 & 0.05819 & 0.06251 & $-204.64(-0.14)$ & 14 & 0.05909 & 0.06348 & $-209.88(-0.15)$ \\
\hline 15 & 0.06251 & 0.06678 & $-200.21(0.17)$ & 15 & 0.06348 & 0.06782 & $-205.57(0.03)$ \\
\hline 16 & 0.06678 & 0.07101 & $-196.63(0.00)$ & 16 & 0.06782 & 0.07211 & $-201.94(-0.21)$ \\
\hline 17 & 0.07101 & 0.07518 & $-193.19(0.11)$ & 17 & 0.07211 & 0.07635 & $-198.11(-0.18)$ \\
\hline 18 & 0.07518 & 0.07930 & $-190.13(0.24)$ & 18 & 0.07635 & 0.08053 & $-195.12(-0.19)$ \\
\hline 19 & 0.07930 & 0.08338 & $-186.23(0.43)$ & 19 & 0.08053 & 0.08467 & $-191.04(-0.38)$ \\
\hline \multirow[t]{2}{*}{20} & 0.08338 & 0.08740 & $-183.40(0.18)$ & 20 & 0.08467 & 0.08876 & $-187.18(-0.23)$ \\
\hline & \multicolumn{3}{|c|}{$w_{\text {DMF }}=0.1509$} & \multicolumn{4}{|c|}{$w_{\text {DMF }}=0.1995$} \\
\hline 2 & 0.00250 & 0.00746 & $-146.45(0.22)$ & 2 & 0.00246 & 0.00734 & $-146.86(-0.90)$ \\
\hline 3 & 0.00746 & 0.01237 & $-231.49(0.285)$ & 3 & 0.00734 & 0.01217 & $-213.90(-2.87)$ \\
\hline 4 & 0.01237 & 0.01723 & $-229.00(1.01)$ & 4 & 0.01217 & 0.01695 & $-211.31(-2.55)$ \\
\hline 5 & 0.01723 & 0.02204 & $-225.22(1.19)$ & 5 & 0.01695 & 0.02168 & $-207.37(-2.15)$ \\
\hline 6 & 0.02204 & 0.02680 & $-222.43(-0.03)$ & 6 & 0.02168 & 0.02636 & $-202.18(-2.18)$ \\
\hline 7 & 0.02680 & 0.03151 & $-218.84(-0.08)$ & 7 & 0.02636 & 0.03099 & $-198.64(-2.09)$ \\
\hline 8 & 0.03151 & 0.03617 & $-214.70(0.16)$ & 8 & 0.03099 & 0.03558 & $-194.67(-2.09)$ \\
\hline 9 & 0.03617 & 0.04078 & $-210.64(0.45)$ & 9 & 0.03558 & 0.04011 & $-191.44(-1.87)$ \\
\hline 10 & 0.04078 & 0.04534 & $-206.19(0.82)$ & 10 & 0.04011 & 0.04460 & $-187.96(-1.78)$ \\
\hline 11 & 0.04534 & 0.04985 & $-202.50(0.72)$ & 11 & 0.04460 & 0.04903 & $-184.91(-1.85)$ \\
\hline 12 & 0.04985 & 0.05431 & $-198.99(0.56)$ & 12 & 0.04903 & 0.05342 & $-180.81(-1.48)$ \\
\hline 13 & 0.05431 & 0.05872 & $-194.81(1.31)$ & 13 & 0.05342 & 0.05776 & $-176.67(-0.46)$ \\
\hline 14 & 0.05872 & 0.06308 & $-194.30(-0.35)$ & 14 & 0.05776 & 0.06205 & $-175.10(-1.68)$ \\
\hline 15 & 0.06308 & 0.06739 & $-189.07(0.45)$ & 15 & 0.06205 & 0.06629 & $-171.40(-1.76)$ \\
\hline 16 & 0.06739 & 0.07166 & $-183.65(1.53)$ & 16 & 0.06629 & 0.07048 & $-168.71(-1.77)$ \\
\hline 17 & 0.07166 & 0.07587 & $-181.03(0.97)$ & 17 & 0.07048 & 0.07462 & $-165.60(-1.71)$ \\
\hline 18 & 0.07587 & 0.08003 & $-177.74(1.31)$ & 18 & 0.07462 & 0.07872 & $-163.24(-1.70)$ \\
\hline 19 & 0.08003 & 0.08414 & $-175.16(0.67)$ & 19 & 0.07872 & 0.08276 & $-160.36(-2.01)$ \\
\hline \multirow[t]{2}{*}{20} & 0.08414 & 0.08820 & $-169.42(2.00)$ & 20 & 0.08276 & 0.08676 & $-155.84(-2.10)$ \\
\hline & & $w_{\text {DMF }}=0.2502$ & & & & $w_{\mathrm{DMF}}=0.2998$ & \\
\hline 2 & 0.00249 & 0.00744 & $-134.15(-4.09)$ & 2 & 0.00230 & 0.00688 & $-87.31(0.28)$ \\
\hline 3 & 0.00744 & 0.01235 & $-200.37(-2.02)$ & 3 & 0.00688 & 0.01140 & $-151.57(-2.50)$ \\
\hline
\end{tabular}


continued Table 2

\begin{tabular}{rccccccc}
\hline$N$ & $m_{N-1} /\left(\mathrm{mol} \cdot \mathrm{kg}^{-1}\right)$ & $m_{N} /\left(\mathrm{mol} \cdot \mathrm{kg}^{-1}\right)$ & $\Delta H\left(m_{N-1} \rightarrow m_{N}\right) /\left(\mathrm{J} \cdot \mathrm{mol}^{-1}\right)$ & $N$ & $m_{N-1} /\left(\mathrm{mol} \cdot \mathrm{kg}^{-1}\right)$ & $m_{N} /\left(\mathrm{mol}^{\prime} \mathrm{kg}^{-1}\right)$ & $\Delta H\left(m_{N-1} \rightarrow m_{N}\right) /\left(\mathrm{J} \cdot \mathrm{mol}^{-1}\right)$ \\
\hline 4 & 0.01235 & 0.01720 & $-199.21(-1.39)$ & 4 & 0.0114 & 0.01589 & $-150.63(-0.57)$ \\
5 & 0.01720 & 0.02200 & $-196.92(-1.33)$ & 5 & 0.01589 & 0.02032 & $-151.81(-2.11)$ \\
6 & 0.02200 & 0.02675 & $-192.51(-1.49)$ & 6 & 0.02032 & 0.02471 & $-151.04(-1.22)$ \\
7 & 0.02675 & 0.03145 & $-188.95(-1.23)$ & 7 & 0.02471 & 0.02905 & $-150.34(-1.33)$ \\
8 & 0.03145 & 0.03610 & $-185.39(-1.16)$ & 8 & 0.02905 & 0.03335 & $-147.65(-0.53)$ \\
9 & 0.03610 & 0.04071 & $-182.35(-1.20)$ & 9 & 0.03335 & 0.03760 & $-145.52(-0.20)$ \\
10 & 0.04071 & 0.04526 & $-179.22(-1.30)$ & 10 & 0.03760 & 0.04181 & $-144.30(-0.99)$ \\
11 & 0.04526 & 0.04976 & $-176.12(-1.00)$ & 11 & 0.04181 & 0.04597 & $-142.09(-0.77)$ \\
12 & 0.04976 & 0.05421 & $-172.34(-1.10)$ & 12 & 0.04597 & 0.05008 & $-138.94(-0.56)$ \\
13 & 0.05421 & 0.05862 & $-170.06(-1.29)$ & 13 & 0.05008 & 0.05415 & $-136.61(-0.11)$ \\
14 & 0.05862 & 0.06297 & $-166.98(-0.99)$ & 14 & 0.05415 & 0.05817 & $-133.77(0.70)$ \\
15 & 0.06297 & 0.06727 & $-163.74(-1.14)$ & 15 & 0.05817 & 0.06215 & $-131.19(0.26)$ \\
16 & 0.06727 & 0.07153 & $-160.90(-1.19)$ & 16 & 0.06215 & 0.06608 & $-129.61(-0.27)$ \\
17 & 0.07153 & 0.07573 & $-157.75(-0.94)$ & 17 & 0.06608 & 0.06996 & $-127.20(-0.22)$ \\
18 & 0.07573 & 0.07988 & $-155.35(-1.13)$ & 18 & 0.06996 & 0.07380 & $-124.63(0.25)$ \\
19 & 0.07988 & 0.08399 & $-151.17(-0.60)$ & 19 & 0.07380 & 0.07759 & $-121.90(0.80)$ \\
20 & 0.08399 & 0.08804 & $-149.77(-1.92)$ & 20 & 0.07759 & 0.08133 & $-119.50(0.66)$ \\
\hline
\end{tabular}

溶剂中 DMF 质量分数 $\left(w_{\mathrm{DMF}}\right)$ 的变化趋势见图 3 .

根据 McMillan-Mayer 理论, 同系焓对作用系数 $h_{\mathrm{xx}}$ 不仅反映了溶质一溶质直接的相互作用, 还反映 了因溶质-溶剂、溶质-共溶剂和溶剂-共溶剂相互 作用的重新分布所引起的各种效应, 是溶剂介入的 溶质-溶质相互作用焓效应的一种度量. ${ }^{15-18}$ 一般认 为, 同系或异系焓对作用系数的大小及符号是由溶 液中各物种间的疏水-疏水、疏水-亲水和亲水-亲 水作用竞争平衡所决定的. $h_{\mathrm{xx}}$ 或 $h_{\mathrm{xy}}$ 为正值, 表明疏 水性作用占优势, 反之则亲水性作用占优势. ${ }^{9,1920}$

实验发现, $\mathrm{L}-\alpha$-氨基丁酸和 $\mathrm{D}-\alpha$-氨基丁酸在纯 水及不同质量分数的 $\mathrm{DMF}+$ 水混合溶剂中的稀释焓 $\Delta H\left(m_{N-1} \rightarrow m_{N}\right)$ 均为负值 (表 1 和表 2), 由此计算出的 相应的同系焓对作用系数 $h_{\mathrm{xx}}$ 均为较大的正值(表

表3 298.15 K 时 L- $\alpha$-氨基丁酸和 D- $\alpha$-氨基丁酸在不同 DMF 质量分数的 DMF+ $\mathrm{H}_{2} \mathrm{O}$ 混合溶剂中焓对作用系数

Table 3 Enthalpic pairwise interaction coefficients of L- $\alpha$-aminobutyric acid and D- $\alpha$-aminobutyric acid in DMF+water mixed solvents with different DMF mass fractions at $298.15 \mathrm{~K}$

\begin{tabular}{ccc}
\hline \multirow{2}{*}{$w_{\text {DMF }}$} & \multicolumn{2}{c}{$h_{\mathrm{xx}} /\left(\mathrm{J} \cdot \mathrm{kg} \cdot \mathrm{mol}^{-2}\right)$} \\
\cline { 2 - 3 } & L- $\alpha$-aminobutyric acid & D- $\alpha$-aminobutyric acid \\
\hline 0 & $431.43( \pm 6.53)$ & $410.19( \pm 2.03)$ \\
0.0515 & $423.44( \pm 4.88)$ & $404.89( \pm 13.2)$ \\
0.1001 & $412.18( \pm 13.6)$ & $392.61( \pm 3.97)$ \\
0.1509 & $376.03( \pm 7.31)$ & $364.26( \pm 7.33)$ \\
0.1995 & $368.11( \pm 9.25)$ & $334.35( \pm 5.65)$ \\
0.2502 & $317.11( \pm 5.89)$ & $305.73( \pm 4.23)$ \\
0.2998 & $283.71( \pm 9.32)$ & $237.23( \pm 5.16)$ \\
\hline \multirow{2}{*}{ The values in parentheses are the evaluated errors: $\pm \sum_{i=1}^{3}\left|h_{\mathrm{xx} i}-\overline{h_{\mathrm{xx}}}\right| / 3$}
\end{tabular}

3), 且随混合溶剂中 DMF 质量分数的增大而逐渐减 少(图 3). 这说明在对分子作用过程中两个 $\alpha$-氨基丁 酸分子 $\alpha$-碳上的乙基 $\left(\mathrm{CH}_{3} \mathrm{CH}_{2}-\right)$ 之间、乙基与 $\mathrm{DMF}$ 分子上的甲基 $\left(\mathrm{CH}_{3}-\right)$ 之间的疏水-疏水作用, 以及 各疏水性基团 $\left(\mathrm{CH}_{3} \mathrm{CH}_{2}-\right.$ 和 $\left.\mathrm{CH}_{3}-\right)$ 与亲水性基团 $\left(-\mathrm{OH} 、-\mathrm{NCHO} 、-\mathrm{COO}\right.$ 和 $\left.-\mathrm{NH}_{3}^{+}\right)$之间的疏水 - 亲 水作用占主导地位, 它们对同系焓对作用系数 $h_{\mathrm{xx}}$ 都 是正的贡献.

众所周知, DMF 分子与水分子之间可以形成分 子间氢键. 由于 $\mathrm{DMF}$ 分子可形成共振结构, 给电子 能力突出, 因此 DMF 和水分子之间形成的氢键比纯 水中水分子本身的氢键强. ${ }^{21-23}$ 在水溶液中, DMF 分 子的氮原子由于两个甲基的保护而不参与氢键形 成, 但酰胺键的氧原子可与水分子形成氢键(图 4).

DMF 与水的氢键属于中等强度的氢键, 它在 本质上是一种静电吸引作用. ${ }^{24}$ 当 $\mathrm{DMF}$ 的质量分数 $w_{\mathrm{DMF}}<0.3$ (物质的量分数 $x_{\mathrm{DMF}}<0.1$ ) 时, 会增强液态水 的有序结构; 当 $0.1<x_{\mathrm{DMF}}<0.7$ 时, 会破坏液态水的有 序结构; 继续增加 $\mathrm{DMF}$ 的浓度 $\left(x_{\mathrm{DMF}}>0.7\right)$ 时, 液态水 的结构又得到增强. ${ }^{20}$ 在我们所研究的混合溶剂组 成范围内 $(w=0-0.3)$, 一方面, 两性溶质 $\alpha$-氨基丁酸 带电的亲水性头基 $\left(-\mathrm{COO}^{-}\right.$和 $\left.-\mathrm{NH}_{3}^{+}\right)$可以破坏 $\mathrm{DMF}+$ 水混合溶剂中水的有序结构; 另一方面, 其 $\alpha-$ 碳上的较强疏水性侧基 $\left(\mathrm{CH}_{3} \mathrm{CH}_{2}-\right)$ 却可以使 $\mathrm{DMF}+$ 水混合溶剂中水的结构更加有序. 但由于 $\mathrm{CH}_{3} \mathrm{CH}_{2}-$ 基团的体积较大, 可以刺破两个带电头基的溶剂化 壳层, 因此与溶剂水之间的疏水水合效应以及与共 溶剂DMF 分子基团之间的疏水-疏水、疏水-亲水作 

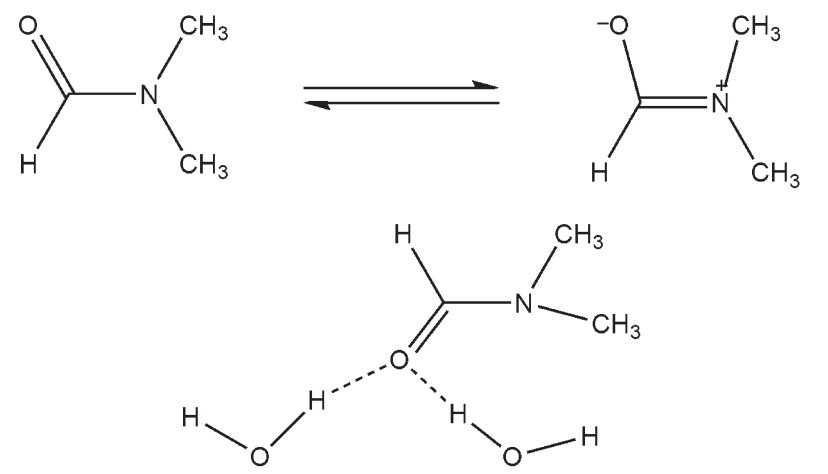

图 4 DMF 的共振结构及其与水分子的氢键

Fig.4 Resonance forms of DMF and its hydrogen bonding with water

用都十分显著; 同时, 在两个 $\alpha$-氨基丁酸两性离子以 “肩并肩”相互作用的优择构型下, $\mathrm{CH}_{3} \mathrm{CH}_{2}$ 一侧基之 间的疏水一疏水作用也十分强烈, 这些因素都表明该 三元溶液中溶质的对分子作用过程以疏水性作用占 主导, 稀释时需释放较大的热量以维持低摘状态, 从 而使得焓对作用系数成为较大的正值..$^{25}$ 随着混合溶 剂中 DMF 浓度的增大, 水结构被不断破坏而使有序 性逐渐降低, ${ }^{21}$ 氨基酸两性离子极性头基之间的离 子-离子作用及其与溶剂分子间的离子-亲水作用逐 渐增强, 体系内亲水性作用的影响逐渐显示出来, 从 而使得焓对作用系数逐渐减小. ${ }^{26,27}$

概括起来, 我们认为在 $\alpha$-氨基丁酸 + 水 $+\mathrm{DMF}$ 三 元溶液体系中, 与共溶剂 DMF 有关的影响溶质焓对 作用系数大小的主要因素有以下几种: (a) $\alpha$-氨基丁 酸分子的非极性乙基与 DMF 分子的两个非极性甲 基之间存在疏水一疏水作用, 对 $h_{\mathrm{xx}}$ 有正的贡献; (b) $\alpha$-氨基丁酸分子的非极性乙基与 DMF 分子的极性 羰基之间存在疏水-亲水作用, 对 $h_{\mathrm{xx}}$ 有正的贡献; (c) $\alpha$-氨基丁酸分子的两个极性头基与 DMF 分子的 两个非极性甲基之间存在亲水一疏水作用, 对 $h_{\mathrm{xx}}$ 有 正的贡献; (d) $\alpha$-氨基丁酸分子的两个极性头基与 DMF 分子的极性羰基之间存在亲水-亲水作用, 对 $h_{\mathrm{xx}}$ 有负的贡献; (e) 在 DMF 浓度较高时, DMF 分子 的羰基氧原子与水分子之间的氢键作用导致水结 构的逐渐破坏以及 $\alpha$-氨基丁酸两性离子的部分去水 化, 对焓对作用系数有负的贡献.

实验还发现, 在相同质量分数组成的 $\mathrm{DMF}+$ 水 混合溶剂中, $\mathrm{L}-\alpha$-氨基丁酸的焓对作用系数值普遍 大于 $\mathrm{D}-\alpha$-氨基丁酸的值(图 3). 这是很有趣的实验现 象, 在其它手性小分子溶液体系中我们有类似发 现, 例如在 DMSO+水混合溶剂中的一些氨基酸和
手性二元醇. ${ }^{11-13}$ 在以纯水作溶剂的二元和三元体系 中, 人们早已发现一些 $\alpha$-氨基酸和手性二元醇对映 异构体的同系和异系焓对作用系数 $\left(h_{\mathrm{L}} 、 h_{\mathrm{DD}} 、 h_{\mathrm{LD}}\right)$ 也 存在较大的差异, 并提出了 “优先构型” 模型以解释 这一实验现象. ${ }^{29-32}$ 综合这些研究结果, 我们认为这 种手性识别或手性区分现象只有在侧链之间以同 手性或异手性构型(LL、DD 或 LD)相互作用时才可 以检测出焓效应来, 而其显著性则受到对映异构体 分子中各功能区域的疏水-疏水、疏水-亲水和亲 水-亲水作用竞争平衡的制约. 在本文实验体系中, 在 $\alpha$-氨基丁酸的同种对映体发生对分子作用时, L-L 分子对比 D-D 分子对在缔合构型上应更有利 于 $\alpha$-碳上疏水侧链 $\left(\mathrm{CH}_{3} \mathrm{CH}_{2}-\right)$ 的靠拢, 彼此疏水基 团的水合壳层发生交盖, 局部受到破坏而释放出一 部分结构化的水, 过程自发、吸热且伴随显著熵增 $(\Delta G<0, \Delta H>0, \Delta S=(\Delta H-\Delta G) / T>0)$, 因而在稀释时释 放出更多热量, $h_{\mathrm{xx}}$ 具有较大的正值.

\section{4 结 论}

用等温滴定量热法测定了 $\mathrm{L}-\alpha$-氨基丁酸和 $\mathrm{D}-\alpha$-氨基丁酸两种对映异构体分别在不同质量分数 $\left(w_{\mathrm{DMF}}=0-0.3\right)$ 的 $\mathrm{DMF}+$ 水混合溶剂中的稀释焓. 依据 McMillan-Mayer 理论求得它们的焓对作用系数. 两 种对映异构体的焓对作用系数值均随着混合溶剂 中 DMF 含量的增大而减小, 并且发现在相同组成的 $\mathrm{DMF}+$ 水混合溶剂中, $\mathrm{L}-\alpha$-氨基丁酸的焓对作用系 数值都要大于 $D-\alpha$-氨基丁酸的值. 实验结果说明 ITC 可以用来区分对映异构体的焓对作用. 强极性 共溶剂 DMF 的介入对两分子溶质缔合过程中各种 疏水性和亲水性相互作用的竞争平衡影响较大, 焓 对作用系数随混合溶剂组成的变化与三元体系中 水结构的改变紧密相关.

\section{References}

(1) McMillan, W. G.; Mayer, J. E. J. Chem. Phys. 1945, 13, 276. doi: $10.1063 / 1.1724036$

(2) Kozak, J. J.; Knight, W. S.; Kauzmann, W. J. Chem. Phys. 1968, 48, 675. doi: 10.1063/1.1668700

(3) Piekarski, H. Pure Appl. Chem. 1999, 71 (7), 1275. doi: 10.1351/ pac199971071275

(4) Castronuovo, G.; Elia, V.; Perez-Casas, S.; Velleca, F. J. Mol. Liq. 2000, 88 (2), 163. doi: 10.1016/S0167-7322(00)00151-3

(5) Zhu, Y.; Yu, L.; Pang, X. J. Chem. Eng. Data 2009, 54 (6), 1910. doi: $10.1021 /$ je900086b 
(6) Palecz, B.; Dunal, J.; Waliszewski, D. J. Chem. Eng. Data 2010, 55 (11), 5216. doi: 10.1021/je100756z

(7) Alston, J. R.; Overson, D.; Poler, J. C. Langmuir 2012, 28 (1), 264. doi: 10.1021/la203765j

(8) Castronuovo, G.; Elia, V.; Moniello, V.; Velleca, F.; PerezCasas, S. Phys. Chem. Chem. Phys. 1999, 1 (8), 1887.

(9) Palecz, B. J. Am. Chem. Soc. 2002, 124 (21), 6003. doi: 10.1021/ ja011937i

(10) Palecz, B. J. Am. Chem. Soc. 2005, 127 (50), 17768. doi: $10.1021 / \mathrm{ja} 0544071$

(11) Zhang, H.; Hu, X.; Shao, S. J. Chem. Eng. Data 2010, 55 (2), 941. doi: $10.1021 / \mathrm{je} 9005322$

(12) Guo, A.; Hu, X.; Fang, G.; Shao, S.; Zhang, H. J. Chem. Eng. Data 2011, 56 (5), 2489. doi: 10.1021/je101353r

(13) Guo, Z.; Hu, X.; Fang, G.; Shao, S.; Guo, A.; Liang, H. Thermochim Acta 2012, 534, 51. doi: 10.1016/j.tca.2012.02.004

(14) Fini, P.; Castagnolo, M. J. Therm. Anal. Calorim. 2001, 66 (1), 91. doi: 10.1023/A:1012435631222

(15) Borghesani, G.; Pulidori, F. Can. J. Chem. 1984, 62, 2898. doi: $10.1139 / \mathrm{v} 84-490$

(16) Thompson, P. T.; Davis, C. B.; Wood, R. H. J. Phys. Chem. 1988, 92 (22), 6386. doi: 10.1021/j100333a041

(17) Gaffney, S. H.; Haslam, E.; Lilley, T. H. Thermochim. Acta 1985, 86, 175. doi: 10.1016/0040-6031(85)87046-5

(18) Piekarski, H. J. Chem. Soc. Faraday Trans. I 1988, 84, 591. doi: 10.1039/f19888400591

(19) Bloemendal, M.; Sijpkes, A. H.; Somsen, G. J. Solut. Chem. 1986, $11(1), 81$.
(20) Bloemendal, M.; Somsen, G. J. Am. Chem. Soc. 1985, 107 (12), 3426. doi: $10.1021 / \mathrm{ja} 00298 \mathrm{a} 005$

(21) Lei, Y.; Li, H.; Pan, H.; Han, S. J. Phys. Chem. A 2003, 107, 1574.

(22) Xu, Z.; Li, H.; Wang, C.; Pan, H.; Han, S. J. Chem. Phys. 2006, 124, 244502. doi: 10.1063/1.2206177

(23) Park, S. K.; Min, K. C.; Lee, C.; Hong, S. K.; Kim, Y.; Lee, N. S. Bull. Korean Chem. Soc. 2009, 30 (11), 2595. doi: 10.5012/ bkcs.2009.30.11.2595

(24) Gilli, P.; Bertolasi, V.; Ferretti, V.; Gilli, G. J. Am. Chem. Soc. 1994, 116 (3), 909. doi: 10.1021/ja00082a011

(25) Gallardo, M. A.; Lilley, T. H.; Linsdell, H.; Otin, S. Thermochim. Acta 1993, 223, 41. doi: 10.1016/0040-6031(93)80118-T

(26) Lin, R.; Hu, X.; Ren, X. Thermochim. Acta 2000, 352-353, 31.

(27) Lu, Y.; Xie, W.; Lu, J. Thermochim. Acta 2002, 385, 1. doi: 10.1016/S0040-6031(01)00700-6

(28) Barone, G.; Castronuovo, G.; Vecchio, P.; Elia, V.; Puzziello, S. J. Solut. Chem. 1989, 18 (12), 1105. doi: 10.1007/ BF00647267

(29) Castronuovo, G.; Elia, V.; Velleca, F. J. Solut. Chem. 1995, 24 (12), 1209. doi: 10.1007/BF00972829

(30) Andini, S.; Castronuovo, G.; Elia, V.; Velleca, F. J. Solut. Chem. 1995, 24 (5), 485.

(31) Andini, S.; Castronuovo, G.; Elia, V.; Pignone, A.; Velleca, F. J. Solut. Chem. 1996, 25 (9), 837.

(32) Smirnov, V. I.; Badelin, V. G. J. Solut. Chem. 2008, 37 (10), 1419. doi: 10.1007/s10953-008-9313-z 